\title{
Spinal Motion Segments - I: Concept for a Subject-specific
}

\section{Analogue Model}

Constantinos Franceskides ${ }^{1}$, Emily Arnold ${ }^{1}$, Ian Horsfall², Gianluca Tozzi ${ }^{3}$, Michael C. Gibson $^{4}$, Peter Zioupos ${ }^{1 *}$

1. Musculoskeletal and Medicolegal Research Group, Cranfield Forensic Institute, Cranfield University,

Defence Academy of the UK; Shrivenham, SN6 8LA, UK

2. Impact and Armour Group, Centre for Defence Engineering, Cranfield University, Defence Academy of the UK; Shrivenham, SN6 8LA, UK

3. School of Engineering, University of Portsmouth, Portsmouth, PO1 3DJ, UK

4. Centre for Simulation \& Analytics, Cranfield University, Defence Academy of the UK; Shrivenham, SN6 8LA, $U K$

*Corresponding author: P.Zioupos

E-mail: p.zioupos@hotmail.co.uk 


\begin{abstract}
Most commercial spine analogues are not intended for biomechanical testing, and those developed for this purpose are expensive and yet still fail to replicate the mechanical performance of biological specimens. Patient-specific analogues that address these limitations and avoid the ethical restrictions surrounding the use of human cadavers are therefore required. We present a method for the production and characterisation of biofidelic, patientspecific, spine motion segment (SMS = 2 vertebrae and the disk in between) analogues that allow for the biological variability encountered when dealing with real patients. Porcine spine segments (L1-L4) were scanned by computed tomography, and 3D models were printed in acrylonitrile butadiene styrene (ABS). Four biological specimens and four ABS motion segments were tested, three of which were further segmented into two vertebral bodies (VBs) with their intervertebral disc (IVD). All segments were loaded axially at $0.6 \mathrm{~mm} / \mathrm{min}$ (strainrate range $\left.6-10 \times 10^{-4} \mathrm{~s}^{-1}\right)$. The artificial VBs behaved like biological segments within the elastic region, but the best two-part artificial IVD were $\sim 15 \%$ less stiff than the biological IVDs. High-speed images recorded during compressive loading allowed full-field strains to be produced. During compression of the spine motion segments, IVDs experienced higher strains than VBs as expected. Our method allows the rapid, inexpensive and reliable production of patient-specific 3D-printed analogues, which morphologically resemble the real ones, and whose mechanical behaviour is comparable to real biological spine motion segments and this is their biggest asset.
\end{abstract}

Keywords: spine, bone analogue, micro-CT, 3D printing, digital image correlation (DIC) 


\section{Introduction}

Patient-specific analogues are needed in the modern fields of forensic and injury biomechanics $[1,2,3]$ because human cadaver specimens are variable and difficult to preserve for biomechanical testing [4] and their use is subject to stringent ethical considerations. Accordingly, mammalian quadruped spines or spine analogues are used instead [5]. Several spine analogues are currently available, but most are not intended for biomechanical testing. They are used for training, for drilling and implant fixation trials, and to demonstrate the range of motion by handling and manipulation $[1,3,6]$.

Replicating the mechanical behaviour of real spines is particularly important in relation to fixing and testing implants. Standardised tests such as ASTM 1717 simply fix implants on blocks of material typically constructed from ultra-high-molecular-weight polyethylene (UHMWPE) without a 'spine segment' being present in parallel with the construct $[7,8,9]$. These are intended for both static and dynamic implant testing but make no attempt to evaluate the implants under loading in the presence of a spine (matching the in vivo environment) and do not replicate the shape, architecture or geometry of the vertebrae in the experimental design. For example, although standardised test methods have been proposed for the reproducible comparison of the stiffness or strength of implants, it is doubtful whether these are indicative of the in vivo behaviour of the spine.

The biofidelity of the standard testing environments must be improved to allow the comparison of devices for orthopaedic applications [9]. A few commercially available spine analogues are similar both geometrically and biomechanically (only in so far as the range of motion is concerned) to human cadaveric spines. For example, a generic biomechanical spine model (Sawbones, USA) is available as a full section (T12-sacrum), a small section (L2-L5) or a single motion segment (L3-L4). However, in these the vertebral body (VB) is a smooth block and as for the bone no effort is made to replicate its internal structure [3]. Spinal implants have previously been usefully tested in conjunction with these models as an alternative to human or animal cadavers [8]. Its advantages include the low variability of the model properties and the long testing life, but limiting factors include the cost, lead time (process time), and lack of patient specificity. In addition, any destructive testing such as the installation of implants cannot be reversed. There is also a commercial option to order a patient-specific analogue, but it only replicates the spine shape and form, not its properties, and of course this is even more expensive. A more accessible spine analogue is needed to (i) 
make biomechanical testing readily available, (ii) at a low cost, (iii) provide reproducible samples and a number of them, (iv) be bespoke for a patient (patient specific), and (v) biofidelic as far as load response is concerned; for all these advantages the answer may provided by using $3 \mathrm{D}$ printing $[2,10,11]$.

The analogue model proposed in this paper is patient-specific and was produced from microCT (computed tomography) data by producing a 3D model of the vertebrae in acrylonitrile butadiene styrene (ABS). The intervertebral disc (IVD) was constructed using topographical data from the endplates. Once the distance between the endplates was determined, liquid polyurethane was injected to form the IVD. Both the biological spinal motion segment and its man-made analogue counterpart were mechanically tested in parallel in axial compression. The stiffness was produced in the linear elastic region from data from virtual and actual extensometers. Surface displacements and strains were determined by digital image correlation (DIC) analysis for each segment. The present approach proposes a methodology using accessible protocols and equipment to create a novel 3D-printed analogue spinal motion segment model, which can easily be applied in future research projects focusing on the prediction and modelling of bone behaviour, either on its own or in conjunction with implants, and in both healthy and diseased conditions.

\section{Materials and methods}

\subsection{Biological specimens}

A porcine spine (from a specimen less than 12 months old, intended for the food supply) was obtained from a local butcher. Four motion segments (L1-L4), i.e. two VBs with their adjoining IVDs from the lumbar region, were prepared from this material. Porcine spine samples were chosen because they are already deemed suitable substitutes for human cadavers and are similar both in geometric and biomechanical properties [12,13]. From the fresh spine, we measured the Shore hardness [14] of an intact IVD (lateral to medial) and a sectioned IVD (superior to inferior), giving values of $63.2(14,8.4)$ and $72.7(10,7.4)$, respectively. This led to the choice of PT Flex 70 (Polytek Development Corp., USA), a twopart polyurethane with a Shore A hardness of 70, as the silicone IVD simulant. 
Three motion segment samples were sectioned to each produce two VB samples and one IVD sample, and one motion segment was left whole (Fig. 1). Part of the pre-CT sample preparation involved placing samples in a water bath to remove any external tissue.

Biological VBs 1-4 were maintained at $40^{\circ} \mathrm{C}$ for $90 \mathrm{~min}$, which has little to no effect on bone properties and thus does not compressive stiffness [15,16,17]. Biological VBs 5 and 6 were immersed at $\sim 80^{\circ} \mathrm{C}$ for $16 \mathrm{~h}$ to determine if there was a noticeable change in bone properties.

IVDs were sectioned within the endplate to ensure the disc was intact. All unnecessary tissue was then removed. The superior and inferior planes of the IVD and VB samples were then ground on a polisher with constant cooling to produce parallel surfaces for mechanical testing. The whole and sectioned motion segment samples were then scanned $(0.0412 \mathrm{~mm}$ at $70 \mathrm{kV}$ and $90 \mu \mathrm{A}$ ) using a XT H225 CT scanner (Nikon Metrology UK Ltd, UK) and reconstructed using CT Pro 3D (Nikon Metrology UK Ltd).

\subsection{Analogue preparation}

All analogue components were printed on a uPrint SE 3D printer (Stratasys Inc., USA) using ABSplus-P430, a production-grade thermoplastic ABS. The vertebral sections were imported to the printer software using Stratasys CatalystEX v4.5 (Stratasys Inc.) as a stereolithographic file (.stl) which was then modified by importing the CT volume file (.vol) into ScanIP (Synopsys Inc., USA) and manipulating the file. Specifically, the background data were duplicated and resampled from 32-bit to 8-bit in an effort to reduce the file size. The applied threshold was based on the distinct histogram peaks and included small trabeculae while minimising soft tissue. All values were selected in order to maintain the morphology while reducing the number of elements and any errors in the resulting model.

After the .stl was generated, an ABS sample described hereafter as an analogue motion segment (AMS) was printed for each real (biological) motion segment (RMS). An additional sample was produced from RMS 4, designated AMS 4ii. The origins of all segments, both biological and analogue, are summarised in Table 1. The setting used for model interior was 'solid' and support fill 'basic'. Any support materials deposited during the printing process were removed manually and with the use of a support cleaning apparatus (PADT Inc., USA) combined with WaterWorks P400SC (Stratasys Inc.).

Earlier tests conducted on 3D-printed ABS cubes revealed their compressive strength. These data showed that the layer orientation influenced the elastic modulus by less than $5.4 \%$ from 
maxima to minima, yet when comparing similar orientations the effect was negligible $[18,19]$. Because all the ABS samples in this study were printed and loaded in the same orientation, we assumed there was a no significant difference in stiffness due to the directionality of the ABS layers.

The IVD of each AMS was formed from PT Flex 70. The design priority for the analogue IVD was to use a suitable rubber compound which, by adjusting its constitution, could be matched to the properties of biological IVD initially on the basis of its Shore A hardness value. Inevitably, this kind of rubber analogue would only provide a uniform layer because the inner design and fibrous architecture of natural IVDs (with their woven collagen fibre layout) is too complex to replicate. This was also true when considering the nucleus pulposus; this being a mucoid protein that allows for distribution of forces. Due to its complex structure and nature, but also the difficulty in printing graded multi moduli materials on a single run, the IVD was replicated as a single phase module with a uniform loading response.

PT Flex 70 was chosen because of its rapid curing time and the Shore A hardness value which matched that of an IVD. An individualised cast was built around the superior and inferior endplates of the IVDs using Sugru mouldable glue (FormFormForm Ltd, UK). The correct height of the IVD (based on CT reconstructions) was ensured by placing PT Flex 70 struts at three points on the endplates. PT Flex 70 was then prepared and injected into the moulds (Fig. $2)$.

A speckle pattern was applied to all samples for DIC analysis [20-24]. White high-contrast paint was used as a base coat on the ABS samples and a black speckle pattern was then applied manually to all samples, with speckle size ranging from 0.35 to $6.35 \mathrm{~mm}$ in diameter (Fig. 3). The optimal speckle size was $3-5$ pixels [25,26], which was equivalent to $0.78-1.3$ $\mathrm{mm}$. All biological samples were removed from the freezer to thaw $6 \mathrm{~h}$ before testing as previously recommended [27,28].

\subsection{Loading experiments}

An Instron 5567 tensile testing machine (Instron, UK ) fitted with a 10-kN load cell was used to compress each sample at a quasi-static loading rate of $0.6 \mathrm{~mm} / \mathrm{min}$ (strain rate range 6$10 \times 10^{-4} \mathrm{~s}^{-1}$ ). All samples were subjected to a $10-50 \mathrm{~N}$ preload before compression to reduce contact errors [28)]. The top platen featured a spherical joint to further minimise contact 
errors and bending moments, and to ensure consistent loading across the sample. The experimental setup is shown in Fig. 4.

Phantom V1212 and V2010 high-speed cameras (Vision Research Inc., USA) fitted with 50 $\mathrm{mm} \mathrm{f} / 1.4$ lenses (Nikon, Japan) recording at $1000 \mathrm{fps}$ were used to obtain DIC data throughout the loading process. The cameras were positioned $25^{\circ}$ apart and $450 \mathrm{~mm}$ from the sample. Calibration was performed with a simple $175 \mathrm{~mm}$ x $140 \mathrm{~mm}$ panel and ARAMIS software (GOM GmbH, Germany). PCC software (Vision Research, USA) was used to interface with the cameras. The DIC analysis facet size was 9 pixels, with a facet step of 3 pixels for all sections. The small facet step increased the measuring point density and also the computational time required, bringing the analysis time per specimen to more than $120 \mathrm{~min}$. The strain calculation method was selected to match the non-uniform thickness of the specimens. Artificial lighting was provided by four LED light sources (Cree, USA), which produced negligible heat. All the natural light sources within the testing area were covered to produce consistent illumination for all tests. All DIC data collected by the high-speed cameras were analysed using ARAMIS software. Due to the large number of frames, highspeed image data were simplified by selecting one in every 10 images.

We performed 31 tests, 20 on ABS samples and 11 on biological samples. All samples were compressed at $0.6 \mathrm{~mm} / \mathrm{min}$. Each test was constrained to a total displacement calculated based on the height of the sample (Eq. 1). The compression tests on all sections are summarised in Table 2.

total displacement $=$ height of vertebral bodies $* 0.05+$ height of vertebral disc $* 0.1$ (1)

RMS 4 was compressed to $1.75 \mathrm{~mm}$ due to load constraints, and greater compression was unnecessary because a sufficient portion of the elastic region was measured, and the yielding of samples was not required.

\section{Results and discussion}

\subsection{Isolated segments - vertebral bodies}

Compression (load vs displacement) data for each analogue section were compared directly to the corresponding data for each biological section. Yielding and plastic deformation was 
observed in the biological vertebral body (RVB) samples but not the analogue vertebral body (AVB) samples at the tested constraints, as shown in Figs 5 and 6. Stiffness was calculated from the linear elastic region of the curves for all samples. RVB samples had a mean stiffness of 8892.6 N/mm $(\mathrm{N}=6, \sigma=3375.7)$ and AVB samples had a mean stiffness of $9720 \mathrm{~N} / \mathrm{mm}$ $(\mathrm{N}=6, \sigma=2614.7)$.

The two samples left in the hot water bath $\left(\sim 80^{\circ} \mathrm{C}\right)$ for $16 \mathrm{~h}$ (RVB 5 and 6$)$ had a noticeably lower stiffness, probably due to the effect of the high temperature. There is a direct relationship between the loss of collagen and alterations to bone mineral structure caused by the boiling of bovine bone tissue, resulting in a three-fold increase in micrometre-scale porosity and an $18 \%$ reduction in bulk density $[15,16]$. Such an effect would thus reduce the magnitude and breadth of the CT-histogram.

Overall there was a very good one-to-one similarity between each RVB and its synthetic analogue counterpart, as shown for a representative RVB in Fig. 7. The two load/deformation curves followed each other until the start of the plastic region (for the biological samples) after which the analogue specimen tolerated an increasing the load whereas the biological specimen yielded and was crushed. The difference in stiffness in five out of six cases between biological and man-made analogue sample was less than 10\% (Fig.8).

\subsection{Isolated segments - intervertebral disks}

Fig. 9 show the response of intervertebral disks (RVD) and their physical models (AVD). The three real disks behaved similarly and exhibited a J-shaped curve with an ever-increasing stiffness due to the presence of the collagen fibres in the annulus fibrosus. The PT Flex 70 analogue specimens were slightly softer (Fig. 8 shows the envelope of the mean behaviour \pm one SD of three curves) and exhibited the typical first stage of an elastomer load/deformation (F/d) curve. These are S-shaped curves, which show a softening behaviour in the early region and then stiffen up later on. S-shaped and J-shaped curves cannot be made to match each other throughout the whole range of F/d values, only within certain regions. In our case, we chose to match the curves in the initial F/d region, starting with the selection of a compound of similar Shore A hardness, which seemed to work well for loads below $1000 \mathrm{~N}$.

\subsection{Motion segments}


Fig. 10 shows the load/displacement data for two RMSs and their analogue counterparts (two VBs and an artificial IVD in between). The analogues were on the whole softer than the natural motion real segments. For example, the stiffness of RMS 4 was 4585.49 N/mm (2, 262), whereas the mean stiffness of AMS 4_1 and AMS 4ii_1 was 1867.6 N/mm (4, 82.1). The discrepancy between AMS models and the biological segments from which the models were created mainly reflects the imperfect matching of the IVD properties in models and biological specimens. This is because the much lower stiffness of IVDs compared to VBs means that much of the compression strain is concentrated in the IVD regions.

To demonstrate this effect, we used DIC to focus on the strains for VBs, IVDs and the total strain across the whole motion segment (Figs. 11 and 12). This was done via use of markers along the length of the component tested. DIC was used over extensometers as 3D DIC can better react to off plane movements; such the ones experienced in IVD bulging under compression. In addition, the final output was filtered using the Bezier interpolation that uses Bernstein polynomials to weight the points.

Calibration was performed using ARAMIS before the test sequence. The static error was \pm $4.2 \%$ as calculated using Eq. 2.

Static Error $=\frac{(\text { Displacement maximum } \mid \text { Displacement Minimum } \mid)}{\text { Length }}(2)$

DIC was performed on all biological and analogue samples with displacement measured between two points (virtual extensometer). Lines were drawn vertically over each VB (upper and lower) section and IVD section. Three sets of points were chosen on each motion segment: the top and bottom of each VB, the top and bottom of the IVD, and the top and bottom of the entire motion segment. The average major strain of each part was represented within each specific strain stage (capture image). Figs 11 and 12 show representative data obtained for RMS4 and AMS4, with the highest strain on the IVD and the lowest strain on the upper and lower VB. As expected, the total full-field strain was between the maxima and minima.

\subsection{Benefits and drawbacks of the model}

Reliable and inexpensive patient-specific analogues are needed in the fields of forensic and injury biomechanics, but it has been challenging to develop models which are both 
straightforward and accurate. We used micro-CT data to develop analogue models of VBs, IVDs and spinal motion segments and then tested them by compression, comparing like for like. The stiffness of the RVBs and AVBs was similar in magnitude. Each RVB behaved in a similar manner to the corresponding AVB section on an individual basis. However, the AVB samples tended to be less variable than the RVB samples, with standard deviations of 2614.7 $\mathrm{N} / \mathrm{mm}(9720 \pm 2614.7, \mathrm{~N}=6)$ and $3375.7 \mathrm{~N} / \mathrm{mm}(8892.6 \pm 3375.7, \mathrm{~N}=6)$ respectively. This is consistent with previously tested spinal analogues $[9,27,28]$. The higher variability of the RVB specimen probably reflects the fact that biological samples vary in both material properties and structural architectural design. The AVB samples were made from the same grade of industrial material (ABS) with only the structure matching the natural one.

The differences in F/d behaviour between the biological and analogue specimens were more pronounced once the biological specimens were taken beyond the yield point. Whereas the RVB samples yielded, the much stronger AVB samples continued into the elastic region. Shearing was observed in one sample (AVD 1), which resulted in delamination of the disc from the endplate and thus a lower stiffness than AVD 2 and AVD 3. We can deduce that facet-less motion segments tend to be less stiff than the complete comparative segments, as previously reported [29].

Strains over the motion segment samples were taken in four different areas: upper vertebral body (UVB), lower vertebral body (LVB), IVD and Total. The strains over the IVD were measured from the superior to the inferior endplate because the surface layer of high-contrast medium bearing the fiducial marks was prone to delamination/deterioration during the loading of the AVD (analogue IVD). These four strains were plotted for motion segment testing and each section was plotted for all segments. All four strains correlated: LVB and UVB experienced the least amount of strain and the IVD experienced noticeably more strain, with the total strain between these values. Strains measured over the UVB followed a similar trend for both AMS and RMS, which was also true of the LVB measurements (Figs 11 and 12). Strains measured over the IVD were noticeably different, with the AMS samples experiencing significantly more strain than the RMS samples.

Not all micro-CT scans were conducted at the same time and marginal differences in greyscale and, more importantly, the shading correction may therefore be present. These differences may cause minor changes in thresholding values during the manipulation and generation of the .stl files. To reduce the size of the .stl files, resampling the data to $0.1 \mathrm{~mm}$ 
was necessary, as well as several other manipulations described in the methods section. These manipulations affect small morphologies in the samples. A higher-resolution printer might achieve a more detailed representation of the internal structure of the sample, which could generate more accurate results. Furthermore, the moulding of the IVD analogue produced small bubbles within the polyurethane which also may affect the mechanical characteristics of the disc.

Another effect not considered here was the testing of the biological discs under hydrated conditions. Research using sheep vertebrae revealed that the stiffness of ovine IVDs differs significantly when tested in a saline bath environment compared to air alone, with this being true in most loading modes such as torsion, flexion and bending: the IVDs were stiffer in air and more pliant in a saline environment [30].

The quality of DIC was limited by the lenses available because the minimum focus distance produced a large viewing field which was suboptimal for data collection. The high-contrast media applied to the ABS disc samples delaminated in some cases during compression and then folded. This delamination and folding affected how much coverage was received from DIC during the later stages of compression.

In the future, further work should be conducted on the stiffness of the motion segment by varying the polyurethane that makes up the IVD and the construction of the facet joints. Polyurethane with a higher Shore hardness value should produce a motion segment with greater stiffness. Facet joints could be made more realistic by adding a cartilage analogue. If the stiffness of AVD and AMS samples can be improved, the method could be applied to human spinal motion segments with a higher degree of agreement.

\section{Conclusions}

The method described in this article produced artificial spine motion segments (SMS) in which the vertebral body (VB) analogues had stiffness values similar to biological VBs and with the added advantage on no biological variability. The polyurethane material chosen for the IVD analogue was significantly less stiff than the material of the biological IVDs because it was originally chosen to match only the Shore A hardness values. Further work is needed to find a more suitable IVD analogue, allowing the production and validation of more accurate 
SMS analogues. DIC data revealed that the biological and the analogue specimens deformed in the same manner with IVDs naturally deforming more than the VBs because of their lower material stiffness. However, the biological IVDs deformed significantly less than the analogue IVDs because the material analogue for IVD did not match the biological IVD closely. The new methodology proposed here produces a simple SMS analogue with a biofidelic behaviour within the elastic regions and in quasi-static axial compressive loading. Further efforts to precisely match the IVD material (analogue to match the biological) will inevitably expand the scope and the usefulness of the man-made analogue introduced here. This product overall promises to improve our ability to build and use accurate patient-specific models for biomechanical testing.

\section{Acknowledgments}

This work was supported by an internal grant from Cranfield University (CF), a project grant from the Royal Centre for Defence Medicine (RCDM) Birmingham (PZ), and by in-kind support from Vision Research. The authors have no conflicts of interest to declare. We acknowledge the work, skill and expertise offered by Karl Norris in the mechanical workshop of Cranfield University, and Jolyon Cleaves of Vision Research for providing the high-speed cameras. Ethical approval was granted by Cranfield University Research and Ethics committee (CURES) approval reference CURES/1014/2016. This paper is dedicated to one of the authors, Dr Mike Gibson, whose untimely death is a great loss to us all.

\section{Data accessibility}

Data for this manuscript is available through the Cranfield University CORD data depository and preservation system (https://cranfield.figshare.com).

\section{Authors' contributions}

Conceptualisation: CF, PZ, IH, GT; Data curation: CF, EA, IH; Formal analysis: CF, EA, IH, MG; Funding acquisition: CF, PZ; Investigation: CF, EA; Methodology: CF, GT; Project administration: PZ; Resources: CF, IH; Software: CF, MG; Supervision: PZ, IH, GT; 
Validation: CF, GT; Visualisation: CF, GT; Writing (original draft): CF; Writing (review and editing): CF, EA, PZ.

\section{References}

Triantafyllou M S, Triantafyllou G S. An efficient-swimming machine. Scientific American, 1995, 272, 64-70.

1. Jones M, Darwall D, Khalid G, Prabhu R, Kemp A, Arthurs O, Theobald P. Development and validation of a physical model to investigate the biomechanics of infant head impact. For. Science International, 2017, 276, 111-9.

2. Inglis S. 3D Printing in the NHS and healthcare sciences. IPEM Scope, 2016, 25, 10-13.

3. Sawbones. Biomechanical Spine Product Research, [2018-02-05], http://www.sawbones.com/UserFiles/Documents/Product/BioSpine_info.pdf.

4. Wilke H J, Krischack S, Claes L E. Formalin fixation strongly influences biomechanical properties of the spine. Journal of Biomechanics, 1996, 29, 1629-31.

5. Smit T H. The use of a quadruped as an in vivo model for the study of the spine biomechanical considerations. European Spine Journal, 2002, 11, 137-44.

6. Sutradhar A, Park J, Carrau D, Miller M J. Experimental validation of 3D printed patientspecific implants using digital image correlation and finite element analysis. Comp. Biol. Med. 2014, 52, 8-17.

7. ASTM. Standard Test Methods for Spinal Implant Constructs in a Vertebrectomy Model. ASTM International, West Conshohocken, PA, USA, 2015.

8. Wang T, Ball J R, Pelletier M H, Walsh W R. Initial experience with synthetic spinal motion segments: biomechanical assessment of high cycle and implant performance. ORS Annual Meeting, New Orleans, LA, USA, 2014.

9. Domann J P. Development and Validation of an Analogue Lumbar Spine Model and its Integral Components. University of Kansas, Lawrence, KA, USA, 2011.

10. Cantrell J, Rohde S, Damiani D, Gurnani R, Di Sandro L, Anton J, Young A, Jerez A, Steinbach D, Kroese C, Ifju P. Experimental characterization of the mechanical properties of 3D-printed ABS and polycarbonate parts. Conference Proceedings of the Society for Experimental Mechanics Series, 2017, 3, 89-105. 
11. Zou R, Xia Y, Liu S, Hu P, Hou W, Hu Q, Shan C. Isotropic and anisotropic elasticity and yielding of 3D printed material. Composites Part B: Engineering, 2016, 99, 50613.

12. Busscher I, van der Veen A J, van Dieën J H. In vitro biomechanical characteristics of the spine: a comparison between human and porcine spinal segments. Spine, 2010, 35, 35-42.

13. Dath R, Ebinesan A D, Porter K M, Miles A W. Anatomical measurements of porcine lumbar vertebrae. Clinical Biomechanics, 2007, 22, 607-13.

14. Shore A F, Shore C P. Apparatus for measuring the hardness of materials. US Patent US1770045 (A) - 1930-07-08, 1930.

15. Zioupos P, Smith C, Yuehuei A. Factors affecting mechanical properties of bone. In: Yuehuei A, Robert D A (eds) Mechanical Testing of Bone and the Bone-Implant Interface. CRC Press, Boca Raton, FL, USA, 1999, 65-85.

16. Roberts S J, Smith C I, Millard A, Collins M J. The taphonomy of cooked bone: characterising boiling and its physico-chemical effects. Archaeometry, 2002, 44, 485-94.

17. Lott B D, Reece F N, Drott J H. Effect of preconditioning on bone breaking strength. Poultry Science, 1980, 59, 724-25.

18. Franceskides C, 2016. Evaluation of bone excision on occipital area of simulated human skull. $22^{\text {nd }}$ Congress of the European Society of Biomechanics, Lyon, 2016.

19. Franceskides C. Subject specific functional model of hard and soft tissues: Skull and Spine. PhD Thesis, Cranfield Univ., 2018.

20. Hisley D, Gurganus J and Drysdale A. Experimental methodology using digital image correlation to assess ballistic helmet blunt trauma. J Appl Mechanics 2010, 78, 051022 .

21. Vassolera J M, Fancelloa E A. Error analysis of the digital image correlation method. Asoc Argentina de Mecán Comp 2012, 29, 6149-61.

22. Pan B, Lu Z, Xie H. Mean intensity gradient: An effective global parameter for quality assessment of the speckle patterns used in digital image correlation. Optics Lasers Eng 2010, 48, 469-77.

23. Lecompte D, Smits A. Bossuyt S, Sol H, Vantomme J, van Hemelrijck D, Habraken A M. Quality assessment of speckle patterns for digital image correlation. Optics Lasers Eng 2006, 44, 1132-45. 
24. Sutton M A, Orteu J J. Schreier H. Image correlation for shape, motion and deformation measurements. New York: Springer, 2009.

25. Palanca M, Tozzi G, Cristofolini L. The use of digital image correlation in the biomechanical area: a review. International Biomechanics, 2016, 3, 1-21.

26. Palanca M, Marco M, Ruspi M L, Cristofolini L. Full-field strain distribution in multivertebra spine segments: An in vitro application of digital image correlation. Medical Engineering \& Physics, 2018, 52, 76-83.

27. Smeathers J E, Joanes D N. Dynamic compressive properties of human lumbar intervertebral joints: a comparison between fresh and thawed specimens. Journal of Biomechanics, 1988, 21, 425-33.

28. Newell N, Little J P, Christou A, Adams M A, Adam C J, Masouros S D. Biomechanics of the human intervertebral disc: A review of testing techniques and results. Journal of the Mechanical Behavior of Biomedical Materials, 2017, 69, 420-34.

29. Holsgrove T P, Gill H S, Miles A W, Gheduzzi S. The dynamic, six-axis stiffness matrix testing of porcine spinal specimens. The Spine Journal, 2015, 15, 176-84.

30. Costi J, Hearn T, Fazzalari N. The effect of hydration on the stiffness of intervertebral discs in an ovine model. Clinical Biomechanics, 2002, 17, 446-55. 
Table 1 Origins of all biological and analogue segments. RMS = real (biological) motion segment. $\mathrm{AMS}=$ analogue motion segment. $\mathrm{RVB}=$ biological vertebral body. $\mathrm{RVD}=$ biological intervertebral disc. $\mathrm{AVB}=$ analogue vertebral body. $\mathrm{AVD}=$ analogue intervertebral disc.

\begin{tabular}{|l|l|l|}
\hline Motion Segment & Biological segment & ABS segment \\
\hline RMS 1 (L5-L6) & RVB 2 & AVB 1 \\
& RVD 1 & AVB 2 \\
& AVD 1 \\
\hline RMS 2 (L4-L5) & RVB 4 & AVB 3 \\
& RVD 2 & AVB 4 \\
& RVB 5 & AVD 2 \\
\hline RMS 3 (L3-L4) & RVB 6 & AVB 5 \\
& RVD 3 & AVB 6 \\
\hline RMS 4 (L1-L2) & RMS 4 RMS 4 1 & AMS 4 \\
\hline
\end{tabular}


Table 2 Compression data for all sections.

\begin{tabular}{|l|l|l|}
\hline Sections & Nominal Strain & Displacement (mm) \\
\hline IVD & 0.1 & $1.4 \pm 0.1$ \\
\hline VB & 0.05 & $0.95 \pm 0.42$ \\
\hline AMS & Eq. 1 & 2.5 \\
\hline RMS & - & 1.75 \\
\hline
\end{tabular}




\section{FIGURE CAPTIONS}

Fig. 1 Motion segments were studied and optimised in three parts. Tissue samples were sectioned at the levels indicated above to produce three specimens: two vertebral bodies (VBs) and one intervertebral disc (IVD) from each motion segment. (a) Anterior view. (b) Lateral view.

Fig. 2 Moulding of the IVD analogue using PTFlex 70.

Fig. 3 Speckle pattern applied to AMS 4 and AMS 4ii.

Fig. 4 The experimental setup, with cameras V1212 left and V2010 right and all light sources in position.

Fig. 5 Compression (load vs displacement) data for AVB segments.

Fig. 6 Compression (load vs displacement) data for RVB segments.

Fig. 7 Load/deformation curves for RVB1 and RVB1 (its ABS made analogue specimen). The difference in load between the two responses is shown separately in dashed line illustrating that there is similarity of responses within the RVB elastic region.

Fig. 8 Percentage difference in stiffness values between each RVB and their ABS-made analogue counterparts. The dashed lines represent $\pm 5 \%$ stiffness difference, shown for comparison.

Fig. 9 Compression (load vs displacement) data for RVD specimens (brown, green and blue solid traces) and the behaviour envelope AVD mean (red dashed) \pm SD (red dotted) of PT Flex 70 analogue specimens.

Fig. 10 Compression (load / displacement) data for RMS 4, AMS 4 and AMS4ii samples.

Fig. 11 Smoothed line strain of four sections on RMS 4: upper vertebral body (UVB), RMS 4: Disc (IVD, measured endplate to endplate), RMS 4: lower vertebral body (LVB), and RMS 4: Total (measured superior to inferior of motion segment).

Fig. 12 Smoothed line strain of four sections on AMS 4: upper vertebral body (UVB), AMS : Disc (IVD, measured endplate to endplate), AMS 4: lower vertebral body (LVB), and AMS 4: Total (measured superior to inferior of motion segment). 


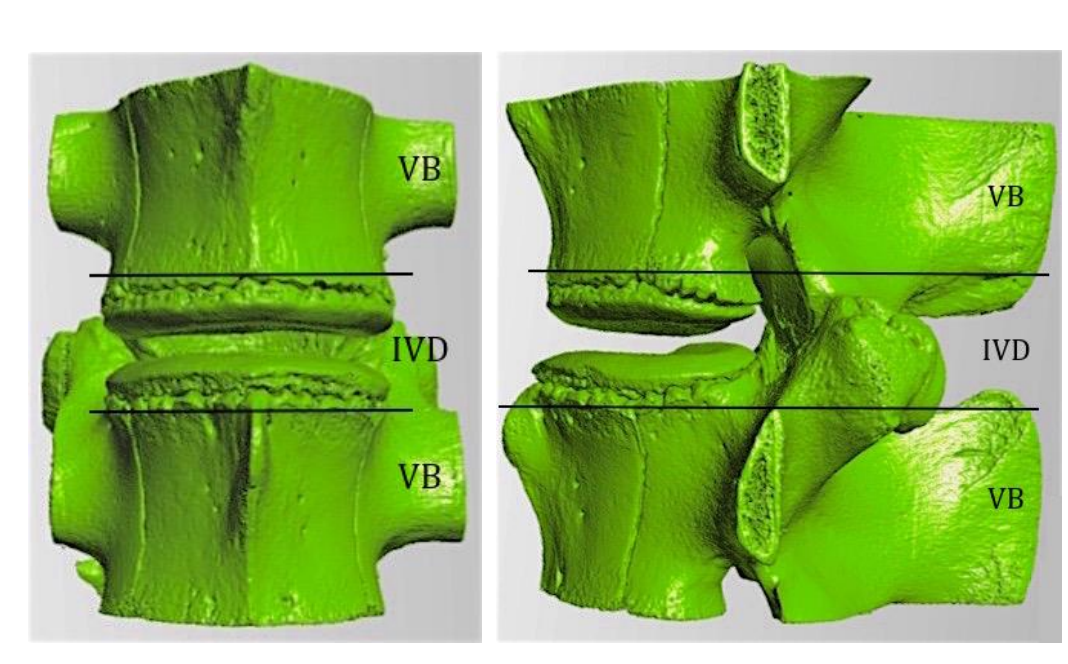

Fig -1

Fig -1 


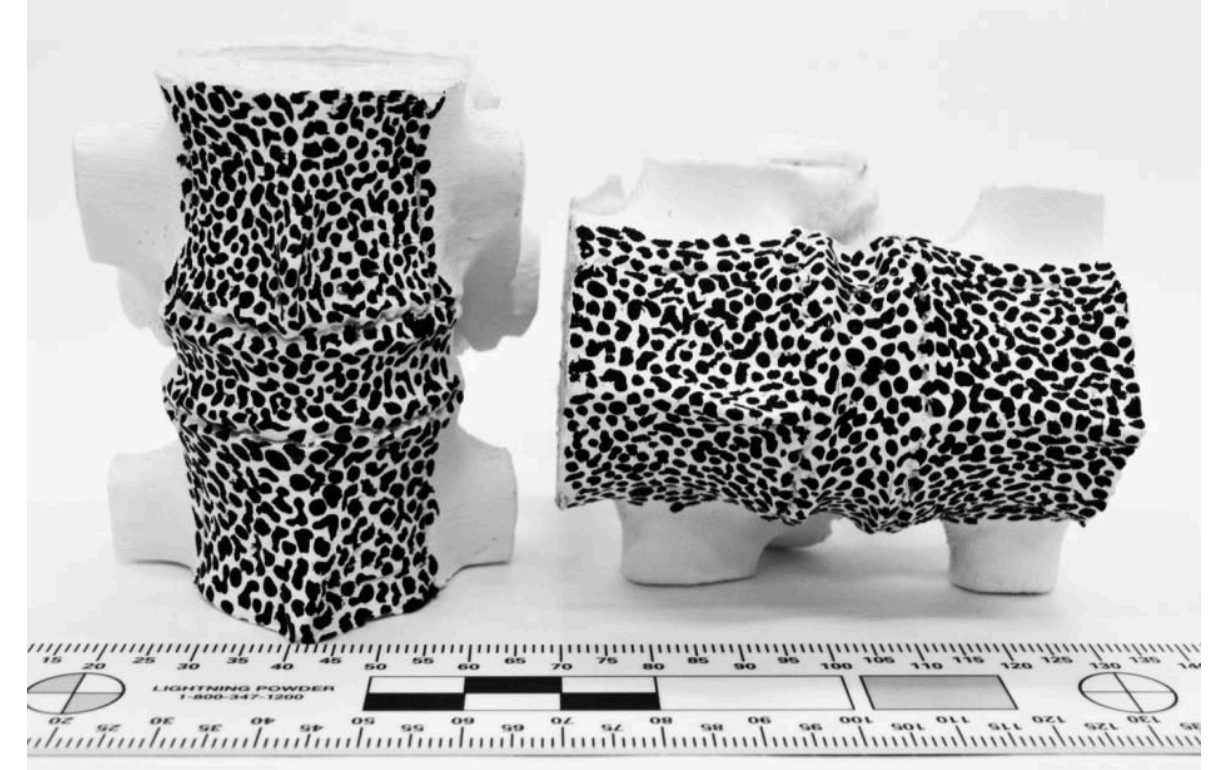

Fig -3

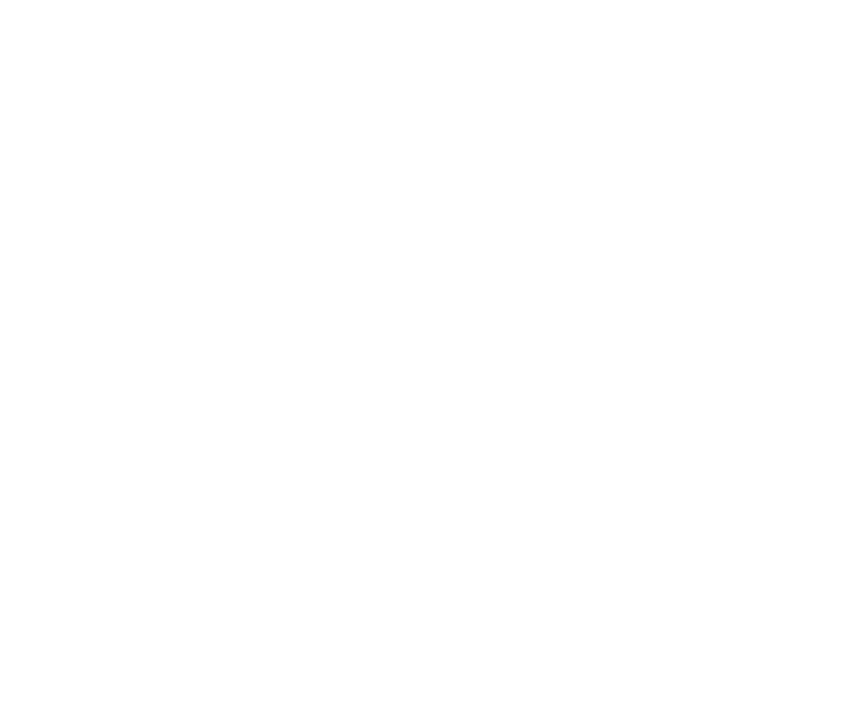

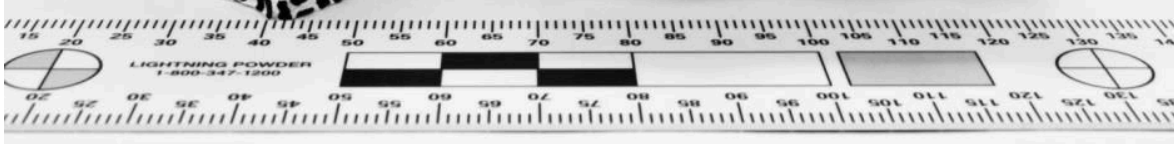

$-3$

o

3.

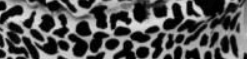

or

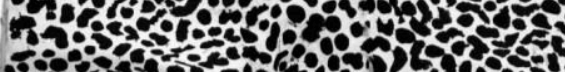

(n)

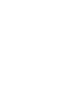




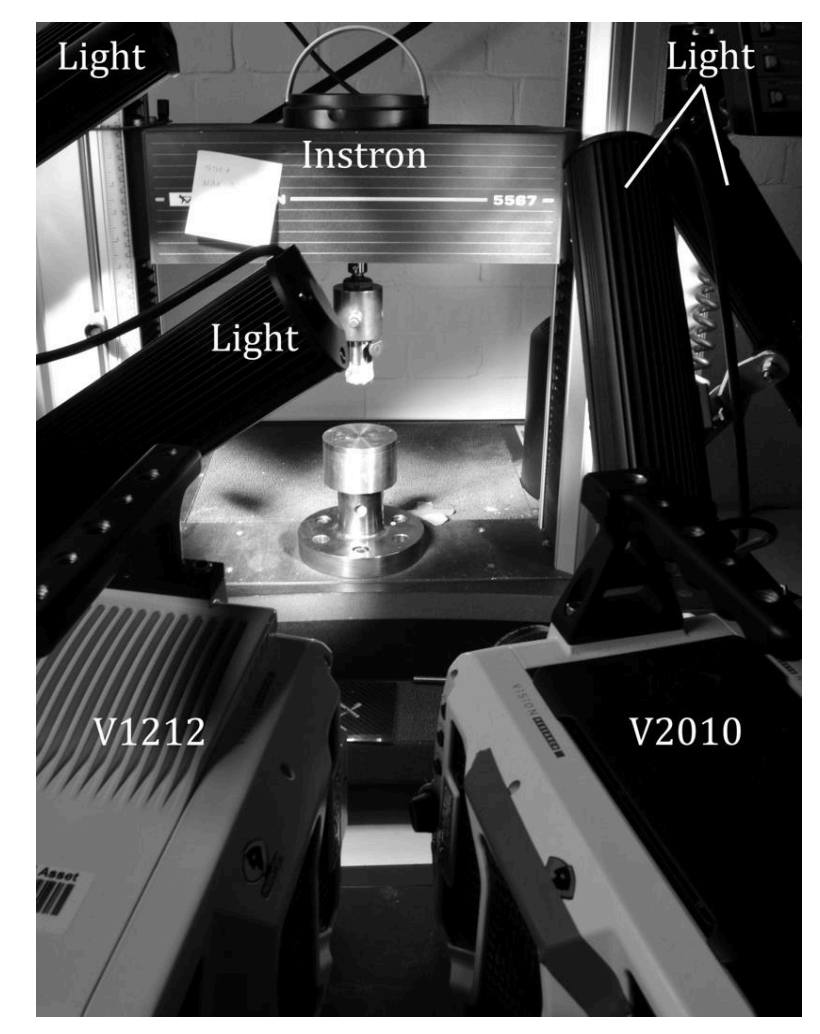

Fig -4

$$
\text { ig -4 }
$$




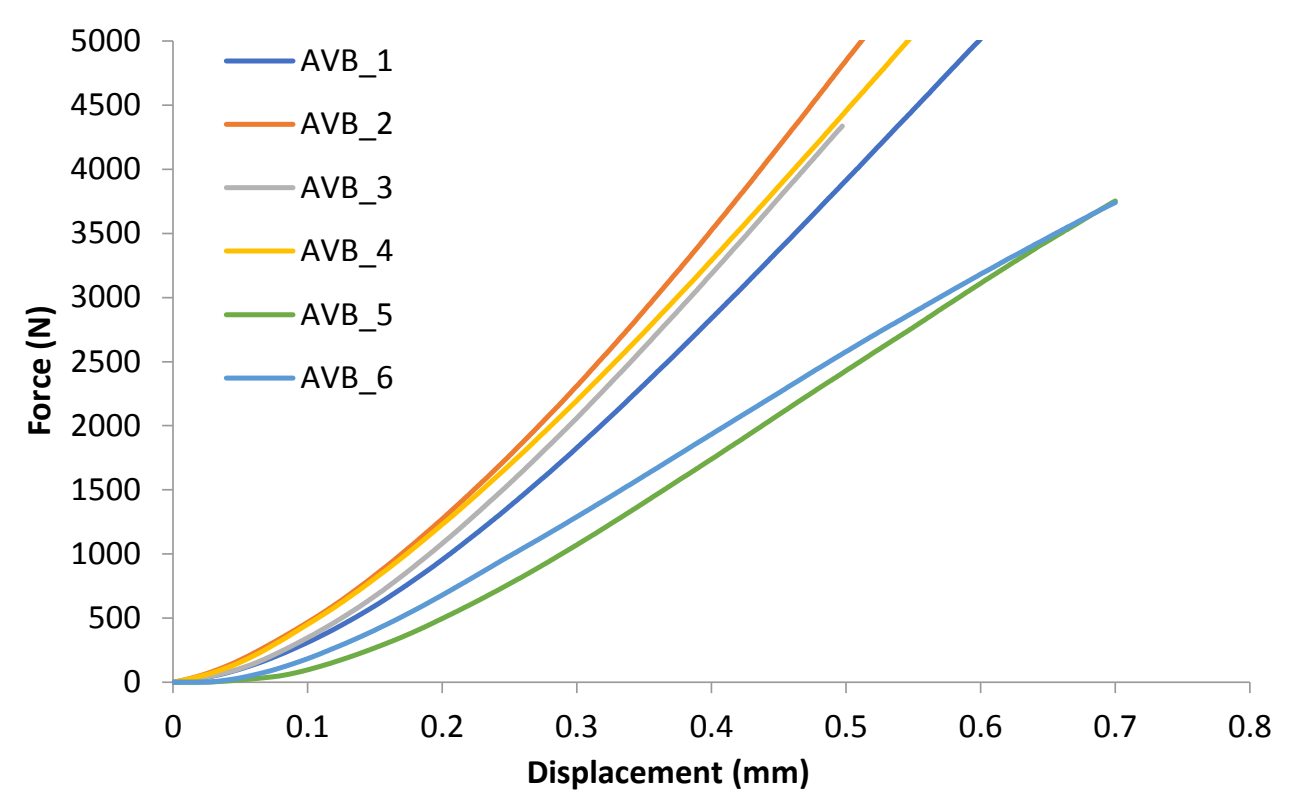

Fig -5 


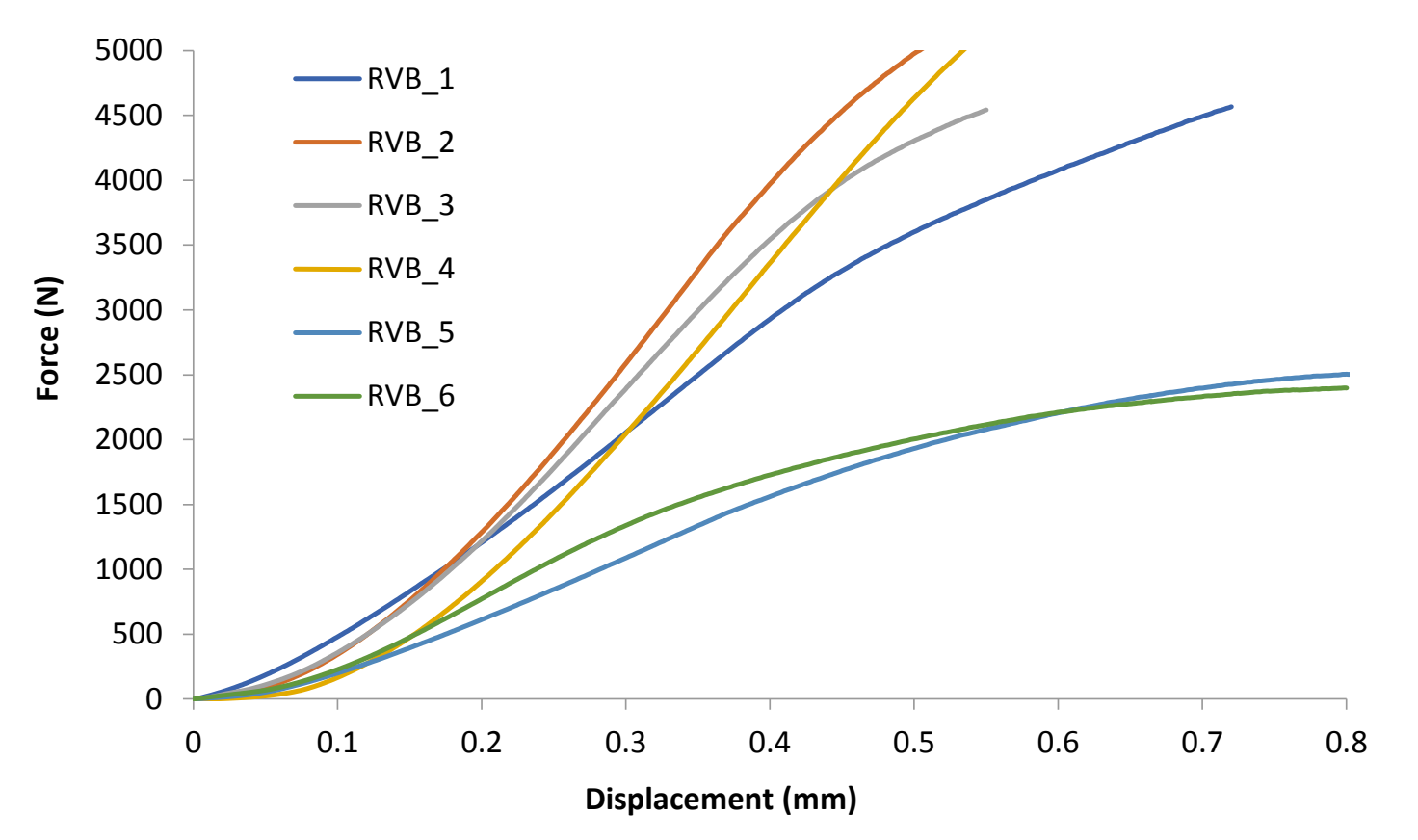

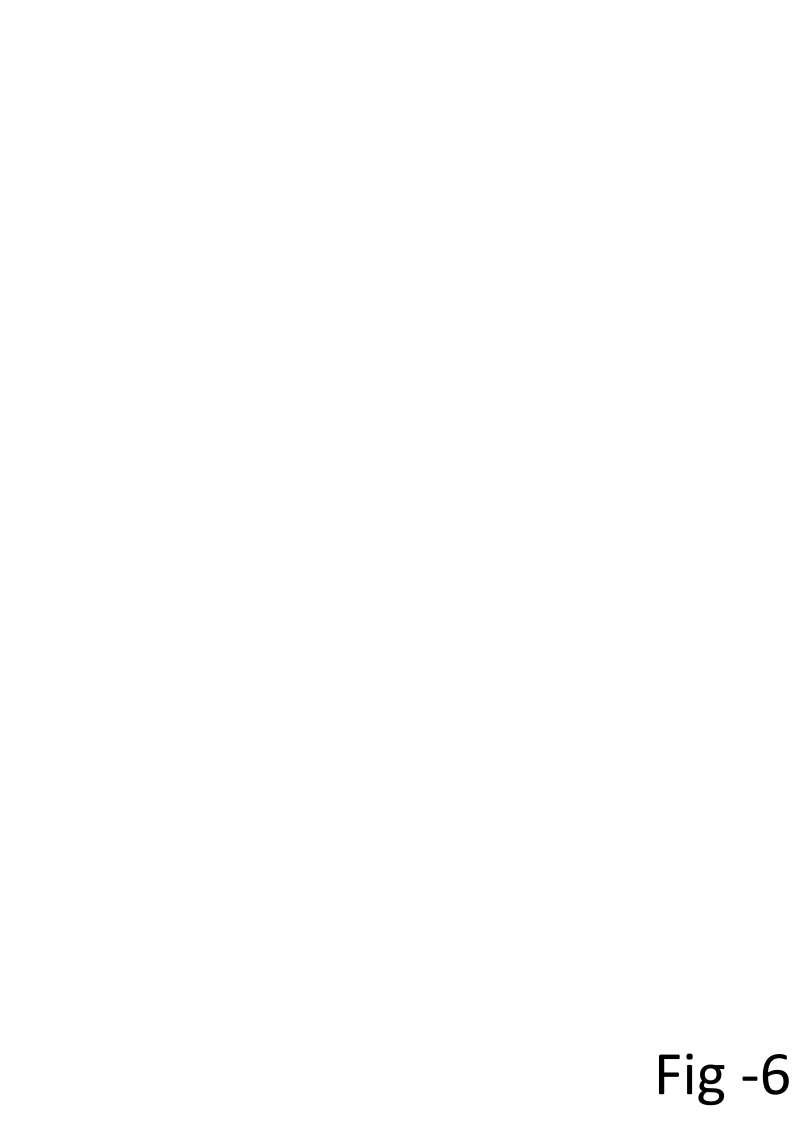

Displacement (mm)

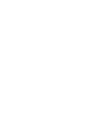




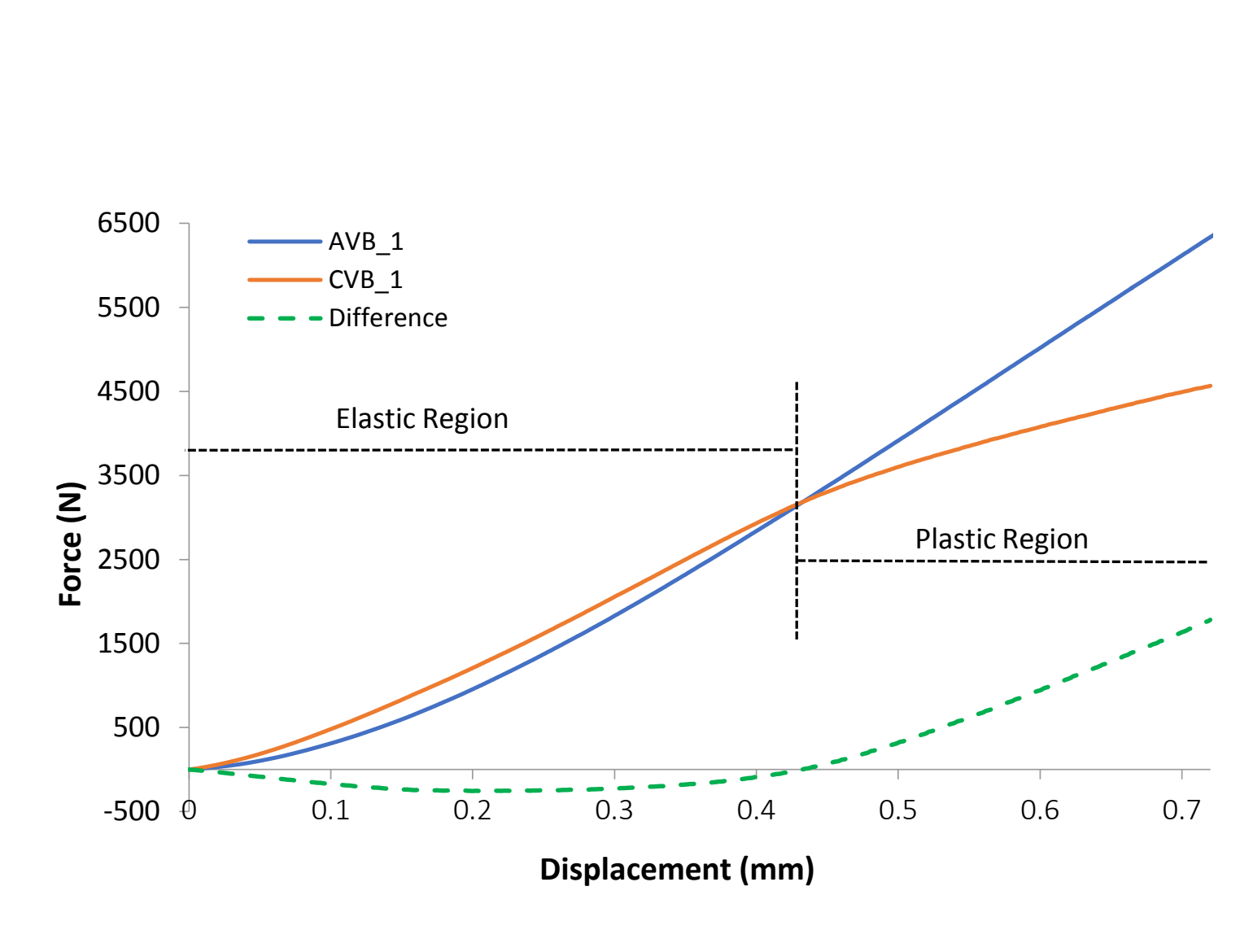

Fig -7

Displacement (mm)

(1)

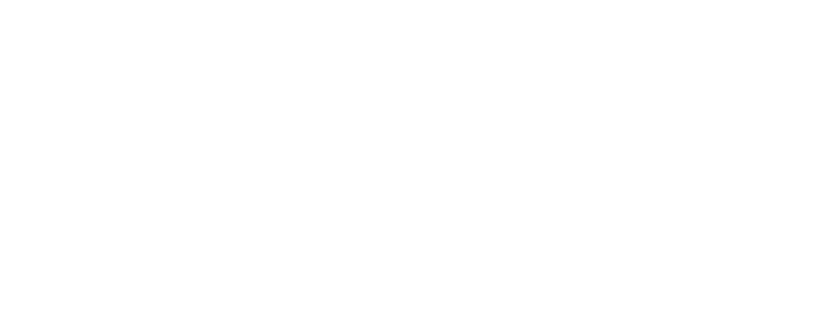

$-7$

(1)

-

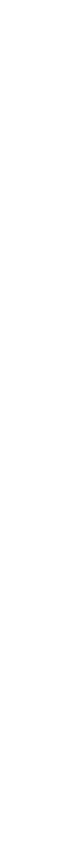

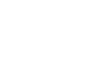




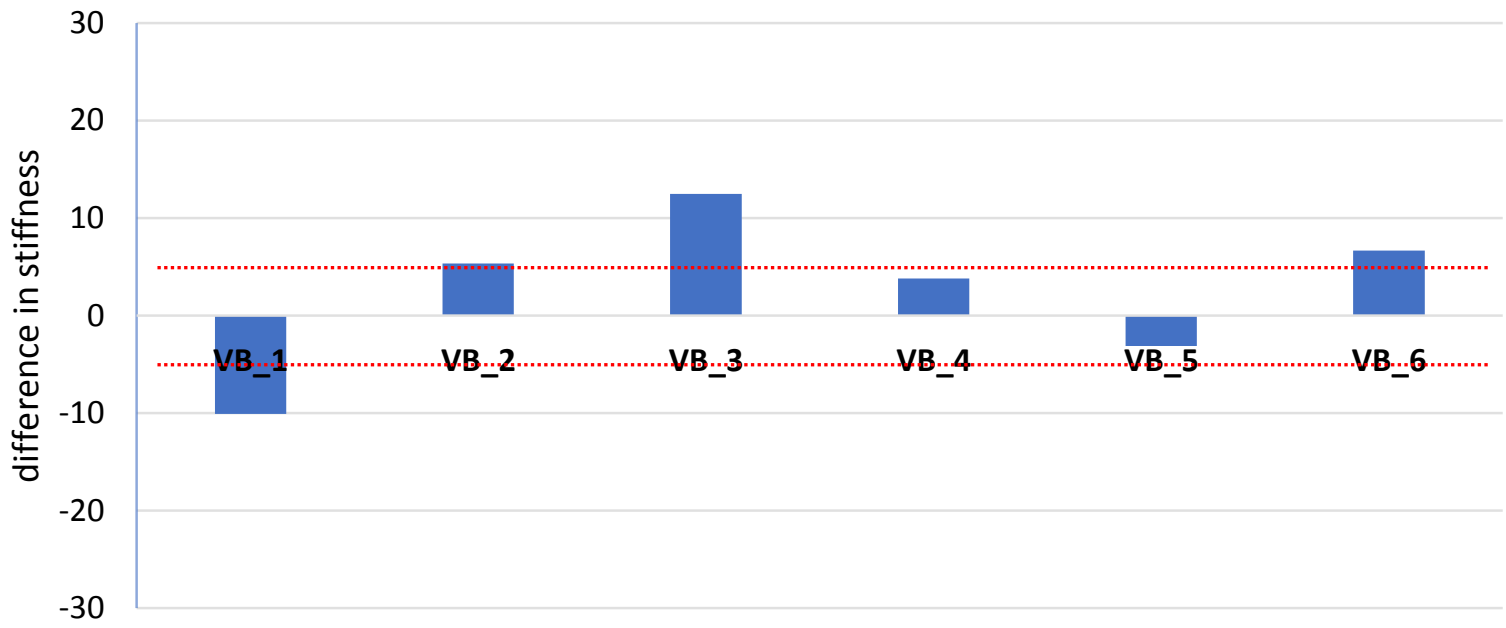

Fig -8

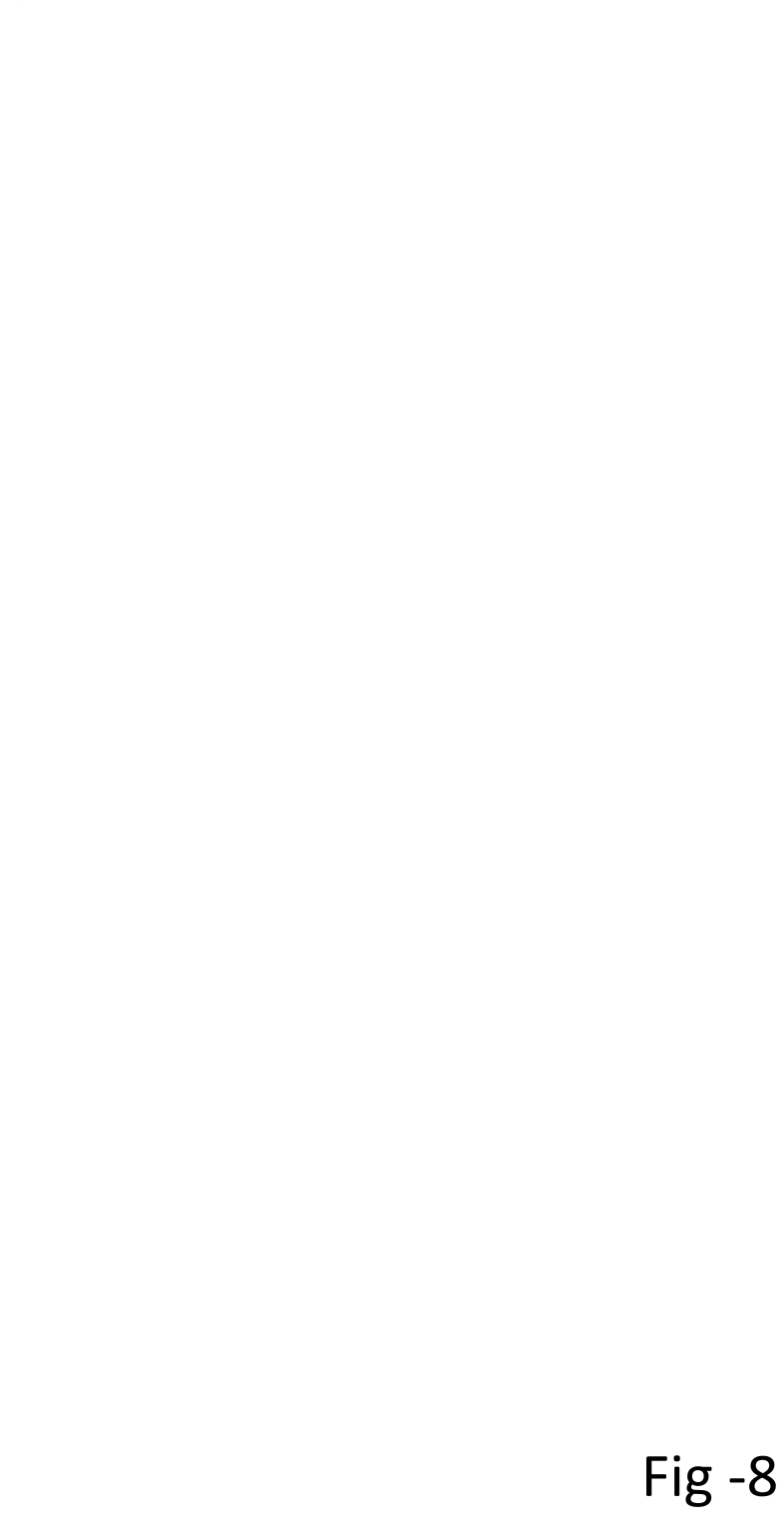
8 


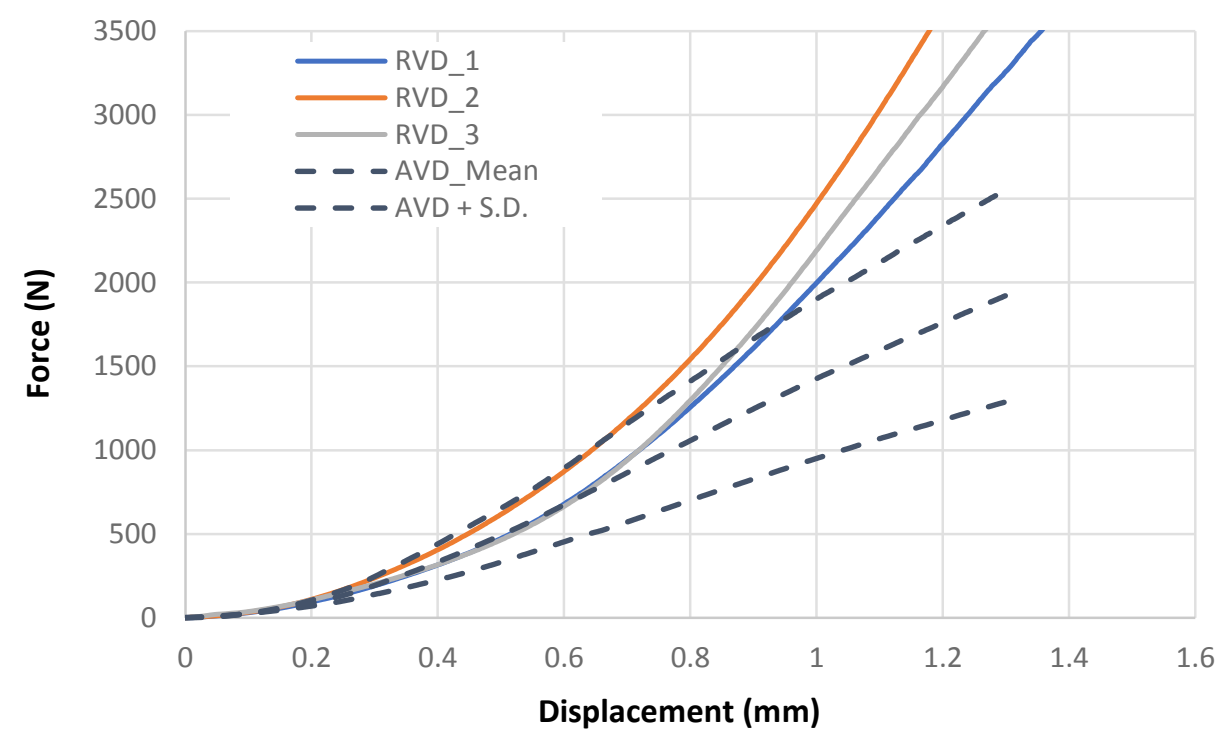

Fig -9

Displacement (mm) 


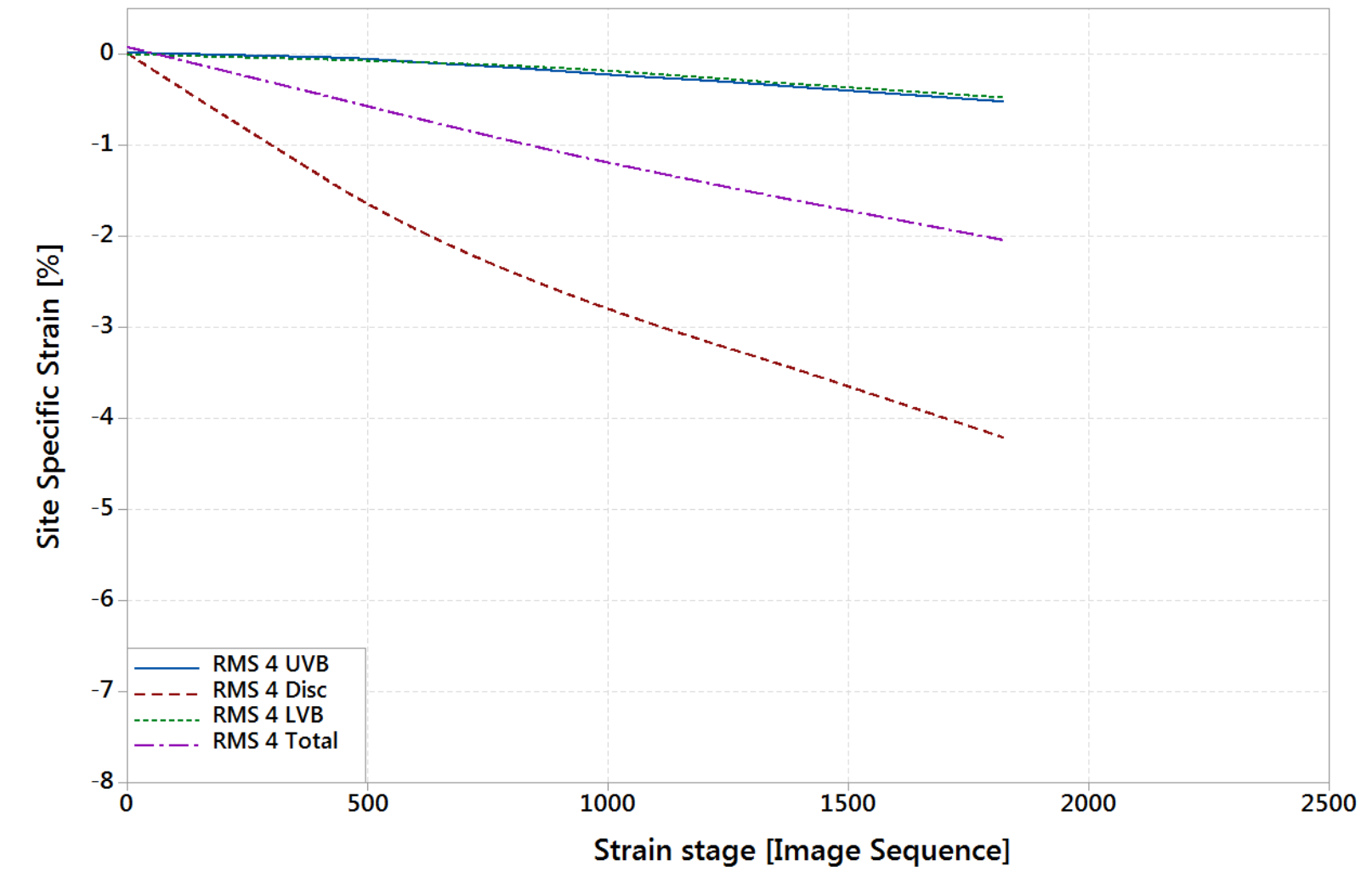

Fig -11

Strain stage [Image Sequence]

1




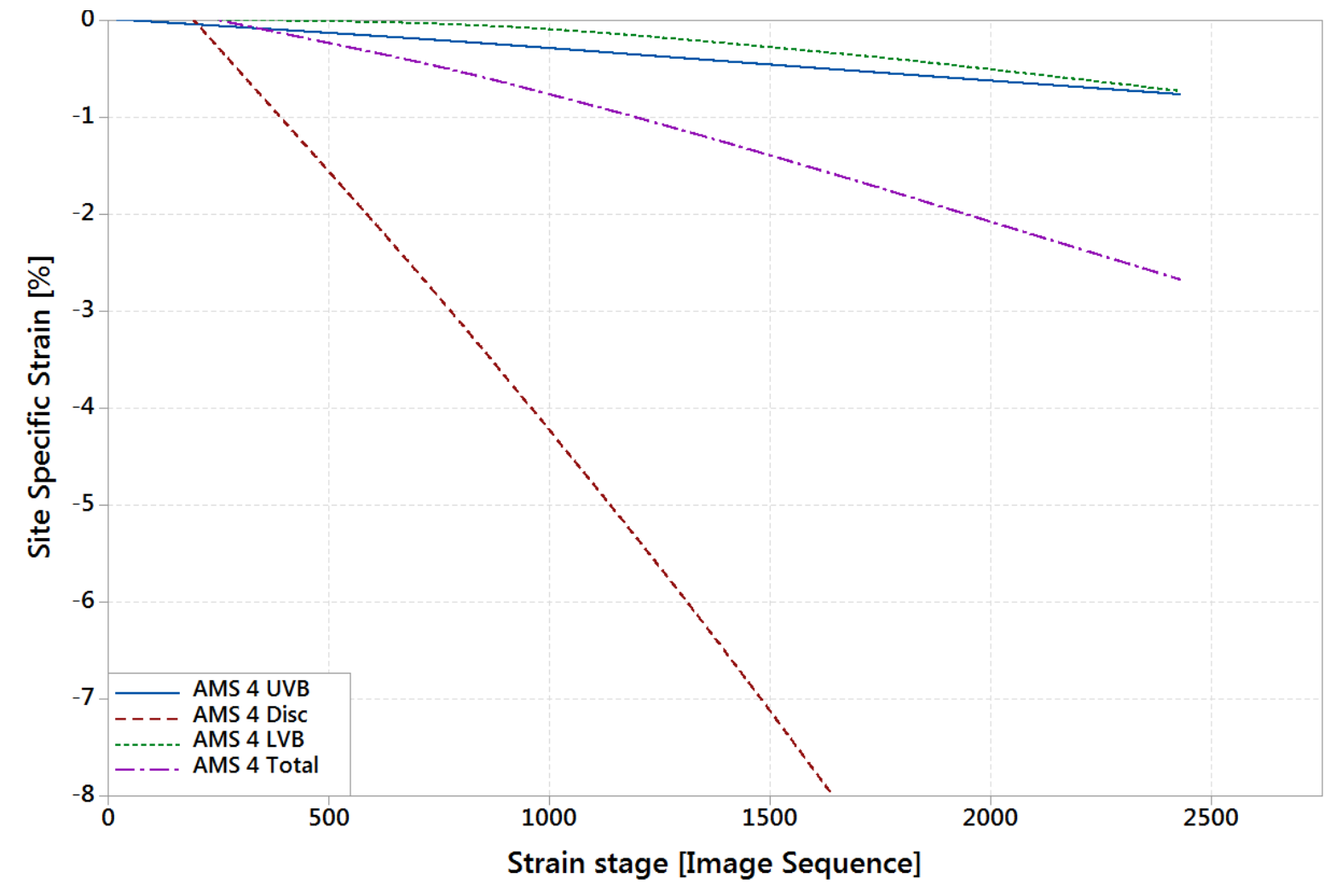

Fig -12

Strain stage [Image Sequence]

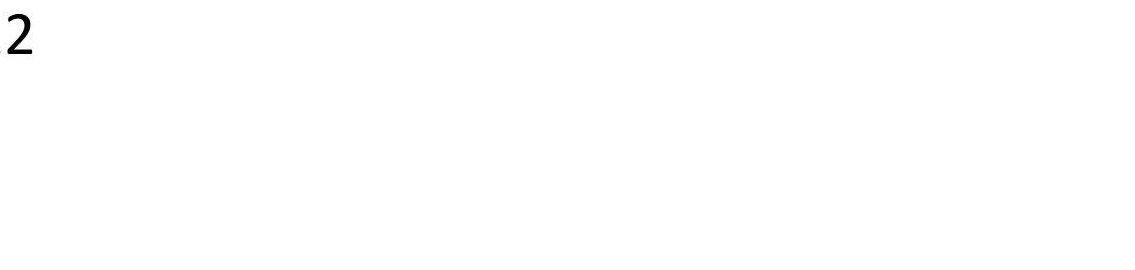

